\title{
Fibroblast growth factors: the keepers of endothelial normalcy
}

\author{
Michael Simons ${ }^{1,2}$ \\ ${ }^{1}$ Yale Cardiovascular Research Center, Department of Internal Medicine and 'Department of Cell Biology, Yale University School of Medicine, New Haven, Connecticut, USA.
}

Endothelial cells (ECs) under physiologic and pathologic conditions are capable of substantial plasticity that includes the endothelial-mesenchymal transition (EndMT). Notably, in the hypoxic pulmonary circulation EndMT likely drives increases in the pulmonary arterial blood pressure, leading to pulmonary arterial hypertension (PAH). However, it is unclear whether suppressing EndMT can prevent PAH development or mitigate established disease. In this issue of the $J C I$, Woo et al. generated mice with EC-specific deletion of FCFR1 and -2 and mice with EC-specific expression of a constitutively active FGFR1 to determine the role of FGF signaling in PAH. Mice with FGFR1/2 deletion in ECs that were exposed to hypoxic conditions developed extensive EndMT and more severe PAH than control mice.

Animals with the constitutively active endothelial FGFR were protected from hypoxia-induced EndMT and PAH development. These findings suggest that FGF signaling may promote vascular resilience and prevent hypoxia-induced development of EndMT and PAH.

\section{Endothelial cell plasticity}

Studies from a number of laboratories have demonstrated a remarkable degree of plasticity of adult endothelial cells (ECs) under physiologic and pathologic conditions. This plasticity ranges from a transient downregulation of EC-specific gene expression to a full disappearance of EC fate marker genes in conjunction with the appearance of genetic programs associated with other cell types, including fibroblasts, smooth muscle cells (SMCs), and macrophages, among others, which is a process termed endothelial-mesenchymal transition (EndMT) (1, 2). Having undergone a partial or full EndMT, ECs acquire the ability to drive a number of pathologic processes, among them inflammation and fibrosis. These changes include secretion of proinflammatory cytokines, expression of adhesion molecules that promote recruitment of circu- lating white blood cells and platelets, and deposition of the extracellular matrix (3). Not surprisingly, such a profound change in EC biology leads to pathological changes that underlie various disease states, including atherosclerosis (4) and cranial cerebral malformations (CCMs) (5). One important disease process recently shown to be driven by EndMT is pulmonary arterial hypertension (PAH), the subject of Woo et al. from the Ornitz lab in this issue of the JCI (6).

The origin and pathophysiologic basis of PAH has long been a contentious and controversial subject. The disease itself is highly heterogeneous, with a variety of genetic, pathologic, and environmental triggers. This variety of causes, notwithstanding the common morphologic basis underlying $\mathrm{PAH}$, is a combination of fibrosis and abnormal SMC coverage of the distal pulmonary arterial tree that leads to

\section{Delated Article: https://doi.org/10.1172/JCl141467}

Conflict of interest: MS is a founder and Chair of the Science Advisory Board of VasoRx, Inc.

increased pulmonary arterial resistance, and, ultimately, an increase in the pulmonary arterial blood pressure. Identification of the source of these new SMCs responsible for the aberrant pulmonary arterial tree muscularization is important to understanding this disease's development. While some studies have clearly shown that preexisting SMCs from proximal parts of the arterial tree proliferate and migrate distally (7), several have suggested that some of the new SMCs are derived from ECs, implying the occurrence of EndMT. Indeed, the presence of EndMT has been documented both in patient tissues and in a mouse model of PAH induced by chronic hypoxia $(8,9)$. With the two potential sources of pathogenic SMC populations thus proposed, the unanswered and critical question is whether suppression of EndMT would prevent PAH development and induce regression of an established disease. The study by Woo et al. (6) in this issue takes an important step in addressing these questions.

\section{A caveat for EC TGF- $\beta$ signaling}

While a number of molecular pathways have been reported as being able to induce EndMT, the principal driver is the activation of endothelial TGF- $\beta$ signaling (1). Remarkably, unlike most cell types where TGF- $\beta$ signaling is antiinflammatory, it is highly proinflammatory in the endothelium (10). This inflammatory quality is an important caveat and underscores the critical importance of endothelium-specific interventions. Activation of EC TGF- $\beta$ signaling can occur as a result of genetic mutations that can directly trigger this process, as observed for example in various CCM syndromes $(11,12)$, or due to increased expression of EC TGF- $\beta$ receptors and increased production/availability of TGF- $\beta$ itself. Since normal EC expression of TGF- $\beta$ receptors (TGF- $\beta$ R 1 and -R2) is very low, increasing receptor expression is central to EndMT. Prolonged exposure to inflammatory cytokines such 


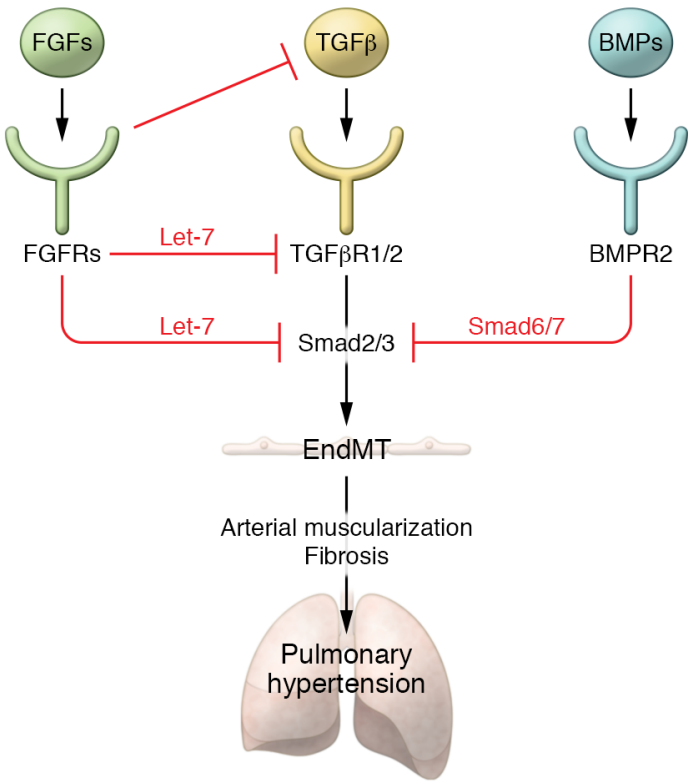

Figure 1. Protective signaling and the development of pulmonary hypertension. Continuous FGF and BMP signaling inputs are required for suppression of the pathogenic endothelial cell TCF- $\beta$ signaling cascade. In the absence of FGF signaling, decreasing let-7 levels allow for increased expression of TCF- $\beta 2$, TCF- $\beta$ R1, and Smad-2, leading to activation of TGF- $\beta$ signaling and induction of EndMT. Constitutively active FGFR1 fully prevents TGF- $\beta$-driven development of pulmonary hypertension.

as IL-1 $\beta$, TNF- $\alpha$, and IFN- $\gamma$, as occurs in the setting of chronic inflammation, results in marked expression of TGF- $\beta$ receptors and ligands, most prominently TGF- $\beta 2$, which can increase in concentration 50- to 100-fold (4). The end result is a proinflammatory environment that sustains continued conversion of ECs to fibroblast- and SMC-like cells, resulting in formation of neointima and fibrosis.

At the molecular level, the key mechanism behind inflammation-induced activation of TGF- $\beta$ receptors and ligand expression is the loss of protective fibroblast growth factor (FGF) signaling due to a decline in EC expression of FGF receptors (FGFRs). This loss in FGF signaling, in turn, leads to an approximately 80- to 100-fold reduction in let-7 miRNA family member levels. Let-7 miRNA family members normally downregulate expression of several key TGF- $\beta$ cascade proteins, including TGF- $\beta$ R1 and Smad-2 (13). The existence of this FGF/TGF- $\beta$ crosstalk and its relevance to EndMT development has been verified in a number of in vitro (13) and in vivo settings, including in patients with atherosclerosis $(4,14-16)$. Studies in that patient population showed a strong correlation between the loss of FGFR1 expression, increase in TGF- $\beta$ R1 expression, activation of TGF- $\beta$ signaling, and the pathologic extent of atherosclerosis (4). The verification of the causal link between these observations is that EC-specific disruption of TGF- $\beta$ signaling dramatically reduces EndMT and essentially eliminates vascular inflammation. The end result is the prevention of atherosclerosis development and extensive (approximately 70\%) regression of fully

The key unverified link in this rather complex pathophysiologic chain is the purported protective role of FGF signaling. To address this issue, Woo et al. generated mice with EC-specific deletion of FGFR1 and -2 and mice with EC-specific expression of constitutively active FGFR1. When subjected to hypoxia, mice with deletion of EC FGFR1/2 developed more severe PAH than control mice, while animals with the constitutively active EC FGFR were protected from PAH development (6). This is a remarkable result that nearly closes the FGF/TGF- $\beta$ crosstalk loop. We now have an in vivo demonstration of the key pathogenic role of EC TGF- $\beta$ activation $(4,14)$; patient data showing an inverse relationship between FGFR1 expression, TGF- $\beta$ activation, and disease severity (4); in vivo demonstration of the protective role of disestablished lesions (10) ruption of TGF- $\beta$ signaling (10); and confirmation of the protective role of EC FGF signaling (6). One caveat here is that studies dealing with disruption of EC TGF- $\beta$ signaling were carried out in atherosclerosis models, whereas the protective effect of FGF signaling was studied in PAH settings. Although it would be important to show that EC TGF- $\beta$ R1 knockout prevents PAH development and constitutively active EC FGFR1 signaling protects from atherosclerosis, it seems safe to assume that the FGF-mediated protection against the TGF- $\beta$ activation paradigm (Figure 1) is fairly universal.

\section{Protective pathways under adverse conditions}

Finally, what is the real biological role of FGFs? As their names suggest, growth factors have traditionally been seen as promoters of cell proliferation and migration. In particular, the first two FGFs discovered, acidic and basic FGFs (FGF1 and FGF2), are potent mitogens; they are capable of inducing proliferation of a number of cell types, including ECs and SMCs (17). Yet, recent studies have begun to suggest that stimulation of cell growth may not be the primary role of these molecules. FGFs act by binding to and activating their tyrosine kinase receptors (FGFRs) alone or in combination with a number of coreceptors. Given the large number of FGF family members and a substantial degree of homology between them, attempts to establish a biological role of the FGF signaling cascade in the vasculature have recently focused on disruption of expression of FGFRs. Surprisingly, although essential during vascular development (18), disruption of FGF signaling in adult mice produced rather mild phenotypes, including a mild increase in baseline permeability and some loss of structural integrity $(19,20)$. However, when subjected to adverse conditions such as ischemia or hypoxia, mice with disrupted EC signaling demonstrate profound abnormalities, including grossly abnormal arteriogenesis and vascular permeability $(21,22)$ as well as the development of EndMT. Taken together with the study of Woo et al. (6), we are left with the suggestion that the main function of FGFs, at least in endothelial biology, is the protection of normalcy under adverse conditions. 
It is important to note that FGF signaling is not the only protective pathway playing a role in PAH development. Genetic mutations affecting bone morphogenic protein receptor II (BMPRII) or abnormal expression of key components of the BMP signaling cascade and various related miRNAs that result in decreased BMP signaling, have all been linked to the development of PAH (23-28). As in the case of FGF/TGF crosstalk, the loss of BMP signaling induces PAH by triggering EndMT. How exactly the EC BMP and FGF protective pathways interact, and whether one can compensate for the other, remains to be determined. Yet, these two strands of evidence point to the importance of vascular resilience in disease prevention (29). There is clearly a critical need to fully understand how such resilience is achieved. Future studies should identify and characterize disease-resistant cell populations (30) and what means exist to restore vascular resilience.

\section{Acknowledgments}

MS is supported in part by NIH grants R01 HL135582 and P01 HL107205 and by the Open Philanthropy Foundation. The author would like to thank MA Schwartz and MJ Mulligan-Kehoe for helpful discussions and P-Y Chen for her key contributions.

Address correspondence to: Michael Simons, YCVRC, 300 George St, Rm 773, New Haven, Connecticut 06510, USA. Phone: 203.737.4643; Email: michael. simons@yale.edu.

1. Dejana E, et al. The molecular basis of endothelial cell plasticity. Nat Commun. 2017;8:14361.

2. Kovacic JC, et al. Endothelial to mesenchymal transition in cardiovascular disease: JACC State-of-the-Art Review. JAm Coll Cardiol.
2019;73(2):190-209.

3. Schwartz MA, et al. A unifying concept in vascular health and disease. Science. 2018;360(6386):270-271.

4. Chen PY, et al. Endothelial-to-mesenchymal transition drives atherosclerosis progression. JClin Invest. 2015;125(12):4514-4528.

5. Maddaluno L, et al. EndMT contributes to the onset and progression of cerebral cavernous malformations. Nature. 2013;498(7455):492-496.

6. Woo KV, et al. Endothelial FGF signaling is protective in hypoxia-induced pulmonary hypertension. J Clin Invest. 2021;131:e141467.

7. Sheikh AQ, et al. Smooth muscle cell progenitors are primed to muscularize in pulmonary hypertension. Sci Transl Med. 2015;7(308):308ra159.

8. Gaikwad AV, et al. Endothelial to mesenchymal transition (EndMT) and vascular remodeling in pulmonary hypertension and idiopathic pulmonary fibrosis. Expert Rev Respir Med. 2020;14(10):1027-1043.

9. Suzuki T, et al. Isolation and characterization of endothelial-to-mesenchymal transition cells in pulmonary arterial hypertension. Am J Physiol Lung Cell Mol Physiol. 2018;314(1):L118-L126.

10. Chen PY, et al. Endothelial TGF- $\beta$ signalling drives vascular inflammation and atherosclerosis. Nat Metab. 2019;1(9):912-926.

11. Castro M, et al. CDC42 deletion elicits cerebral vascular malformations via increased MEKK3-dependent KLF4 expression. Circ Res. 2019;124(8):1240-1252.

12. Fisher OS, et al. Structure and vascular function of MEKK3-cerebral cavernous malformations 2 complex. Nat Commun. 2015;6:7937.

13. Chen PY, et al. FGF regulates TGF- $\beta$ signaling and endothelial-to-mesenchymal transition via control of let-7 miRNA expression. Cell Rep. 2012;2(6):1684-1696.

14. Evrard SM, et al. Endothelial to mesenchymal transition is common in atherosclerotic lesions and is associated with plaque instability. Nat Commun. 2016;7:11853.

15. Souilhol C, et al. Endothelial-mesenchymal transition in atherosclerosis. Cardiovasc Res. 2018;114(4):565-577.

16. Chen PY, et al. Endothelial-to-mesenchymal transition, vascular inflammation, and atherosclerosis. Front Cardiovasc Med. 2020;7:53. 17. Ornitz DM, Itoh N. The fibroblast growth factor signaling pathway. Wiley Interdiscip Rev Dev Biol. 2015;4(3):215-266

18. Yu P, et al. FGF-dependent metabolic control of vascular development. Nature. 2017;545(7653):224-228.

19. Murakami M, et al. The FGF system has a key role in regulating vascular integrity. JClin Invest. 2008;118(10):3355-3366

20. De Smet F, et al. Fibroblast growth factor signaling affects vascular outgrowth and is required for the maintenance of blood vessel integrity. Chem Biol. 2014;21(10):1310-1317.

21. Oladipupo SS, et al. Endothelial cell FGF signaling is required for injury response but not for vascular homeostasis. Proc Natl Acad Sci U S A . 2014;111(37):13379-13384.

22. House SL, et al. Endothelial fibroblast growth factor receptor signaling is required for vascular remodeling following cardiac ischemiareperfusion injury. Am J Physiol Heart Circ Physiol. 2016;310(5):H559-H571.

23. Chaikuad A, et al. Structural consequences of BMPR2 kinase domain mutations causing pulmonary arterial hypertension. Sci Rep. 2019;9(1):18351.

24. Deng Z, et al. Familial primary pulmonary hypertension (gene PPH1) is caused by mutations in the bone morphogenetic protein receptor-II gene. Am J Hum Genet. 2000;67(3):737-744.

25. Happe $\mathrm{C}$, et al. The BMP receptor 2 in pulmonary arterial hypertension: when and where the animal model matches the patient. Cells. 2020;9(6):1422

26. Shintani M, et al. A new nonsense mutation of SMAD8 associated with pulmonary arterial hypertension. J Med Genet. 2009;46(5):331-337.

27. Tielemans B, et al. TGF $\beta$ and BMPRII signalling pathways in the pathogenesis of pulmonary arterial hypertension. Drug Discov Today. 2019;24(3):703-716

28. Liu T, et al. miR-27a promotes endothelialmesenchymal transition in hypoxia-induced pulmonary arterial hypertension by suppressing BMP signaling. Life Sci. 2019;227:64-73.

29. Gao Y, Galis ZS. Exploring the role of endothelial cell resilience in cardiovascular health and disease. Arterioscler Thromb Vasc Biol. 2021;41(1):179-185.

30. Simons $\mathrm{M}$, et al. Resilience, disease and the age of single cell science. Aging (Albany NY) 2020;12(3):2028-2029. 